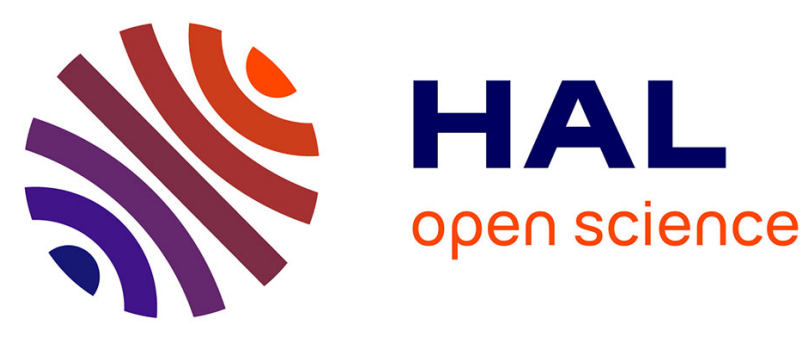

\title{
Yield Design of Periodically Heterogeneous Plates
}

\author{
Jérémy Bleyer, Duc Toan Pham, Patrick de Buhan, Céline Florence
}

\section{To cite this version:}

Jérémy Bleyer, Duc Toan Pham, Patrick de Buhan, Céline Florence. Yield Design of Periodically Heterogeneous Plates. Springer International Publishing. Direct Methods for Limit and Shakedown Analysis of Structures, 220, Springer International Publishing, pp.143-158, 2015, Advanced Computational Algorithms and Material Modelling Series: Solid Mechanics and Its Applications, 978-3-319-12928-0. 10.1007/978-3-319-12928-0_8. hal-01115853

\section{HAL Id: hal-01115853 \\ https://hal-enpc.archives-ouvertes.fr/hal-01115853}

Submitted on 28 Mar 2015

HAL is a multi-disciplinary open access archive for the deposit and dissemination of scientific research documents, whether they are published or not. The documents may come from teaching and research institutions in France or abroad, or from public or private research centers.
L'archive ouverte pluridisciplinaire HAL, est destinée au dépôt et à la diffusion de documents scientifiques de niveau recherche, publiés ou non, émanant des établissements d'enseignement et de recherche français ou étrangers, des laboratoires publics ou privés. 


\title{
Yield design of periodically heterogeneous plates
}

\author{
Jeremy Bleyer ${ }^{1}$ and Duc Toan Pham ${ }^{1,2}$ and Patrick de Buhan ${ }^{1}$ and \\ Céline Florence ${ }^{2}$ \\ 1 : Université Paris-Est, Laboratoire Navier, \\ Ecole des Ponts ParisTech-IFSTTAR-CNRS (UMR 8205) \\ 6-8 avenue Blaise Pascal, Cité Descartes, 77455 \\ Champs-sur-Marne, FRANCE \\ Correspond. author : jeremy.bleyer@enpc.fr \\ 2 : Centre Scientifique et Technique du Bâtiment (CSTB), \\ 84 avenue Jean Jaurès, 77447 Marne-la-Vallée Cedex 2, FRANCE
}

March 28, 2015

\begin{abstract}
This work addresses the determination of the overall strength capacities of periodically heterogeneous plates within a yield design framework. Illustrative applications focus, notably, on reinforced concrete slabs in fire conditions. A homogenization procedure and related numerical tools are proposed to compute macroscopic strength criteria expressed in terms of generalized forces (membrane and bending solicitations). To this end, a yield design auxiliary problem is formulated on the representative threedimensional unit cell and a numerical resolution by a static approach is presented, making use of simple 3D equilibrium finite elements. A particular emphasis is put on the link between the local strength criterion of steel and concrete and the resulting optimization problem, which can be formulated, either as a second-order cone programming (SOCP) problem or, more generally, as a semi-definite programming (SDP) problem. A first illustrative example of a concrete slab with a single array of steel bars will be used to validate the approach. Then, the influence of fire conditions on the strength capacities of reinforced concrete slabs will be investigated and numerical computations will be confronted to experimental results.
\end{abstract}

\section{Introduction}

Direct methods such as yield design or limit analysis of heterogeneous structures can be difficult to perform due to the presence of rapidly and strongly varying material properties on a large scale structure. Despite the efficiency of modern computational techniques, numerical computations on heterogeneous structures are, therefore, out of reach due to the high degree of local refinement needed to correctly capture such variations and provide sufficiently accurate solutions or bounds. To overcome these difficulties, it seems natural to replace the initially heterogeneous 
problem by an equivalent homogeneous one which is likely to be much easier to solve. Homogenization theory in yield design for periodically heterogeneous media, initially developed by Suquet [1] and de Buhan [2], is a proper framework which gives a consistent definition of the strength domain of the equivalent homogeneous medium. It relies on the resolution of an auxiliary yield design problem formulated on the unit cell with specific boundary conditions.

An analytical determination of such macroscopic strength criteria is, in general, limited to simple geometries or macroscopic loadings $[2,3]$. Hence, numerical approaches have to be used to determine the macroscopic strength criterion. Even if elasto-plastic computations can be performed on the unit cell [4], direct limit analysis or yield design computations are more straightforward and easier to perform. This approach has been adopted for porous media $[5,6]$, periodic plates solicited in their own plane $[3,7]$, masonry walls [8] or stone column reinforced soils [9].

Such numerical approaches generally rely on a finite element discretization of the unit cell and the formulation of the auxiliary problem as an optimization problem. Since many yield criteria can be written using conic constraints $[10,11,12]$ (either quadratic or semi-definite cones), the arising convex optimization problem belongs to the category of conic programming. Interior-point solvers, initially designed for linear programming only, have been developed to encompass this broader class of problems and implemented in commercial codes such as the MOSEK software package [13] for instance. Today, these solvers are considered by the scientific community as the most efficient numerical tools for such problems due to their excellent performance in practice.

This work will be devoted to the formulation and numerical resolution of auxiliary yield design problems for periodic plates in membrane and bending solicitations using a conic programming formulation. Section 2 is devoted to the formulation of the problem in a yield design homogenization framework. Section 3 treats the numerical aspects of finite element discretization and conic programming formulation. Finally, some illustrative applications are presented in section 4 and a specific procedure designed to take into account the effect of fire conditions on the macroscopic strength domain, is also discussed.

\section{Homogenization theory in yield design for periodic plates}

\subsection{Initial heterogeneous yield design problem}

We consider a heterogeneous plate of thickness $h$ modeled as a threedimensional continuum $\Omega$, the middle plane of which, denoted by $\omega$, is supposed to lie in the $\left(O, \underline{e}_{1}, \underline{e}_{2}\right)$ plane. The thickness direction is given by the vector $\underline{e}_{3}$ (Figure 1 ).

Assuming that the plate loading depends upon several loading parameters $\underline{Q}$, the domain $K$ of potentially safe loads $\underline{Q}$ is defined, according to the yield design theory $[14,15]$, as the set of loads such that there exists at least one statically admissible (S.A.) stress field $\underline{\underline{\sigma}}(\underline{x})$ satisfying the strength criterion at each point of the plate :

$$
K=\{\underline{Q} \mid \exists \underline{\underline{\sigma}}(\underline{x}) \text { S.A. with } \underline{Q}, \forall \underline{x} \in \Omega \quad \underline{\underline{\sigma}}(\underline{x}) \in \mathcal{G}(\underline{x})\}
$$




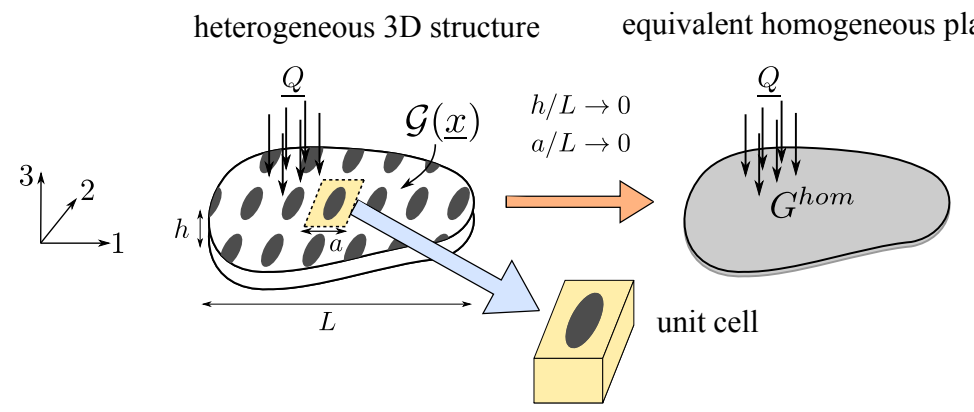

Figure 1: Homogenization procedure for periodic plates

where $\mathcal{G}(\underline{x})$ denotes the convex strength criterion at a point $\underline{x}$ in the plate expressed in terms of the local stress tensor $\underline{\underline{\sigma}}$.

\subsection{Homogeneous yield design plate problem}

The present work deals with plates for which the strength properties are periodic in their in-plane direction, that is, there exists two vectors $\underline{a}_{1}$ and $\underline{a}_{2}$ in $\omega$ such that $\mathcal{G}(\underline{x})$ can be reproduced by periodicity along $\underline{a}_{1}$ and $\underline{a}_{2}$

$$
\mathcal{G}\left(\underline{x}+n_{1} \underline{a}_{1}+n_{2} \underline{a}_{2}\right)=\mathcal{G}(\underline{x}), \quad \forall \underline{x} \in \Omega, \forall n_{1}, n_{2}, \in \mathbb{Z}
$$

The two vectors $\underline{a}_{1}$ and $\underline{a}_{2}$ define the parallelepipedic unit cell of the periodic plate. The natural idea of homogenization theory is to substitute the local heterogeneous strength criterion $\mathcal{G}(\underline{x})$ formulated in terms of local stress tensors by a homogenized or macroscopic strength criterion $G^{\text {hom }}$ with equivalent strength properties formulated in terms of generalized forces of the plate, as illustrated in figure 1. In the following, it will be supposed that the plate is infinitely resistant to shear forces so that the macroscopic strength criterion will be expressed in terms of a membrane force tensor $\underline{\underline{N}}$ and a bending moment tensor $\underline{M}$. In this case, the set of potentially safe loads for the homogenized problem can be defined as :

$$
K^{h o m}=\left\{\underline{Q} \mid \exists \underline{\underline{N}}(\underline{x}), \underline{\underline{M}}(\underline{x}) \text { S.A. with } \underline{Q}, \forall \underline{x} \in \omega \quad \underline{\underline{N}}(\underline{x}), \underline{\underline{M}}(\underline{x}) \in G^{h o m}\right\}
$$

The principal difference with respect to the definition of $K$ is that the stress fields to be considered are now generalized forces consistent with a plate model and defined on the plate middle plane $\omega$ while the strength criterion $G^{\text {hom }}$ is now homogeneous.

The main result of homogenization theory in yield design $[1,2]$ states that both sets $K$ and $K^{\text {hom }}$ becomes identical under an appropriate definition of $G^{\text {hom }}$ and under the hypothesis that the different length scales are separated which reduces here to the following hypotheses :

- $h \ll L$, this hypothesis states that the thickness in the transverse direction of the continuum is sufficiently smaller than the characteristic length $L$ in the plane $\omega$ so that $\Omega$ can be modeled as a thin plate;

- $a \ll L$, this hypothesis states that the typical length scale of the variation of the strength properties in the plane $\omega$ is much smaller 
than the characteristic length of the structure in this plane, so that the initially heterogeneous material can be replaced by an equivalent homogeneous one.

In the case when $h \ll a$, i.e. when the plate thickness is much smaller than the material properties variation length, it is possible to replace the initial heterogeneous 3D continuum by a heterogeneous plate (implicit up-scaling procedure) and perform the homogenization of the strength properties on a plate model directly [16]. In the present work, we will assume that $h \sim a$ so that both up-scaling procedures have to be performed in one step $[17,8,18]$. In particular, the unit cell will be modeled as a 3D continuum.

\subsection{Definition of the macroscopic strength crite- rion from the solution of a yield design auxiliary problem}

The macroscopic strength criterion is defined and computed via the resolution of a specific auxiliary yield design problem formulated on the plate unit cell $A$. Its middle plane is denoted by $\omega_{A}$. The appropriate conditions to formulate an appropriate auxiliary problem in the framework of periodic homogenization theory are the following :

- zero distributed body forces;

- periodic conditions on the lateral boundary of the unit cell and stress-free boundary conditions on the tip and bottom surfaces;

- averaging relation relating the microscopic stress fields to the macroscopic generalized forces.

Hence, we introduce the set $S A(\underline{\underline{N}}, \underline{M})$ of tensor stress fields $\underline{\underline{\sigma}}$ defined on the unit cell which are statically admissible with a macroscopic membrane tensor $\underline{\underline{N}}$ and a macroscopic bending moment $\underline{\underline{M}}$ as follows :

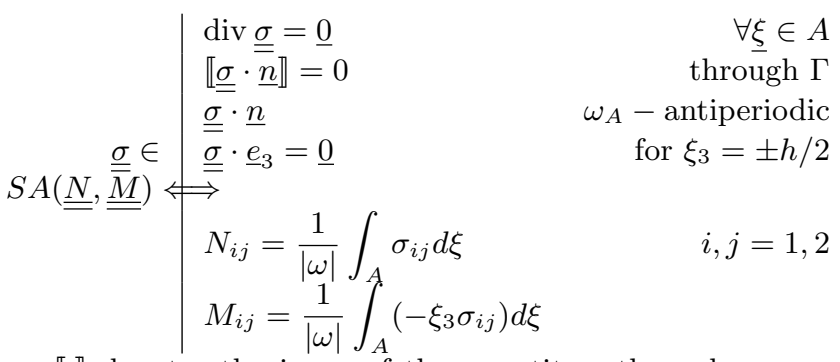

where $\llbracket \cdot \rrbracket$ denotes the jump of the quantity - through any possible discontinuity surface $\Gamma$. Note that the boundary conditions correspond to traction-free conditions on the top and bottom surfaces of the plate, whereas the stress vector $\underline{\underline{\sigma}} \cdot \underline{n}$ should be antiperiodic only on the lateral boundaries of the plate.

Finally, $G^{\text {hom }}$ is defined as the set of all macroscopic membrane forces and bending moments for which there exists such a stress field $\underline{\underline{\sigma}}$, satisfying the local strength criterion every where. That is :

$$
G^{h o m}=\{(\underline{\underline{N}}, \underline{\underline{M}}) \text { s.t. } \exists \underline{\underline{\sigma}} \in S A(\underline{\underline{N}}, \underline{\underline{M}}) ; \underline{\underline{\sigma}}(\underline{\xi}) \in \mathcal{G}(\underline{\xi}) \quad \forall \underline{\xi} \in A\}
$$


Let us remark that a kinematic definition of $G^{\text {hom }}$, through the definition of its support function $\Pi_{h o m}$ where virtual velocity fields are considered, is also possible but will not be considered in this work.

\section{Numerical resolution of the auxiliary problem}

In this section, the auxiliary yield design problem will be solved using a finite element lower bound static approach in association with conic programming.

\subsection{Finite element discretization of the unit cell}

In the following, the unit cell $A$ is discretized using $N_{E}$ tetrahedral equilibrium finite elements. Hence, the six components of the stress tensor are assumed to vary linearly. The values at the 4 vertices are then arranged in a 24 components vector $\vec{\sigma}^{e}=\left\{\sigma_{11}^{(1)}, \sigma_{22}^{(1)}, \ldots, \sigma_{23}^{(4)}\right\}^{T}$.

Due to the linear variation of $\underline{\underline{\sigma}}$, the equilibrium equation (4) is exactly satisfied by enforcing it at only one point inside each element $A_{e}$ through a $3 \times 24$ matrix $\vec{D}^{e}$ :

$$
\operatorname{div} \underline{\underline{\sigma}}=\underline{0} \quad \forall \underline{\xi} \in A_{e} \Longleftrightarrow \vec{D}^{e} \vec{\sigma}^{e}=\overrightarrow{0}
$$

The continuity equation (5) is enforced at each vertex of a face shared by two adjacent elements $e$ and $e^{\prime}$ using a $9 \times 48$ matrix $\vec{C}^{e, e^{\prime}}$ :

$$
\llbracket \underline{\underline{\sigma}} \cdot \underline{n} \rrbracket=\underline{0} \quad \forall \underline{\xi} \in A_{e} \cap A_{e^{\prime}} \Longleftrightarrow \vec{C}^{e, e^{\prime}}\left\{\begin{array}{c}
\vec{\sigma}^{e} \\
\vec{\sigma}^{e^{\prime}}
\end{array}\right\}=\overrightarrow{0}
$$

The enforcement of the periodic boundary condition (6) on the lateral boundary $A_{\text {lat }}$ can be expressed in a similar way :

$$
\underline{\underline{\sigma}} \cdot \underline{n}^{+}+\underline{\underline{\sigma}} \cdot \underline{n}^{-}=\underline{0} \quad \forall \underline{\xi} \in A_{l a t} \Longleftrightarrow \vec{P}^{e, e^{\prime}}\left\{\begin{array}{c}
\vec{\sigma}^{e} \\
\vec{\sigma}^{e^{\prime}}
\end{array}\right\}=\overrightarrow{0}
$$

whereas the traction-free condition on the top and bottom surfaces is written as :

$$
\underline{\underline{\sigma}} \cdot \underline{n}=\underline{0} \quad \forall \xi_{3}= \pm h / 2 \Longleftrightarrow \vec{S}^{e} \vec{\sigma}^{e}=\overrightarrow{0}
$$

The averaging relations are computed as follows :

$$
\begin{gathered}
N_{i j}=\frac{1}{|\omega|} \int_{A} \sigma_{i j} d \xi \Longleftrightarrow \vec{N}=\left\{\begin{array}{l}
N_{11} \\
N_{22} \\
N_{12}
\end{array}\right\}=\underbrace{\left[\begin{array}{lll}
\overrightarrow{A_{N}} & \ldots & \overrightarrow{A_{N}}
\end{array}\right]}_{\overrightarrow{A_{N}}}\left\{\begin{array}{c}
\vec{\sigma}^{1} \\
\vdots \\
\vec{\sigma}^{N_{E}}
\end{array}\right\} \\
M_{i j}=\frac{1}{|\omega|} \int_{A} \sigma_{i j} d \xi \Longleftrightarrow \vec{M}=\left\{\begin{array}{l}
M_{11} \\
M_{22} \\
M_{12}
\end{array}\right\}=\underbrace{\left[\begin{array}{lll}
\overrightarrow{A_{M}} 1 & \ldots & \overrightarrow{A_{M}}
\end{array}\right]}_{\overrightarrow{A_{M}}}\left\{\begin{array}{c}
\vec{\sigma}^{1} \\
\vdots \\
\vec{\sigma}^{N_{E}}
\end{array}\right\}
\end{gathered}
$$


All the equilibrium, continuity and boundary condition constraints are finally assembled into a global constraint matrix $\vec{C}$ such that

$$
\boldsymbol{\Sigma} \in S A(\vec{N}, \vec{M}) \Longleftrightarrow \begin{array}{r}
\vec{C} \boldsymbol{\Sigma}=\overrightarrow{0} \\
\vec{N}=\overrightarrow{A_{N}} \boldsymbol{\Sigma} \\
\vec{M}=\overrightarrow{A_{M}} \boldsymbol{\Sigma}
\end{array}
$$

where $\boldsymbol{\Sigma}=\left\{\begin{array}{c}\vec{\sigma}^{1} \\ \vdots \\ \vec{\sigma}^{N_{E}}\end{array}\right\}$ collects all stress components. It is to be noted that, since all equations are exactly satisfied, the previous relation defines a subset of the set of statically admissible stress fields.

\subsection{Fulfillment of the strength criterion using conic constraints}

In the present work, illustrative examples are concerned with reinforced concrete slabs so that the local strength criterion will either be a von Mises criterion (for steel) or a Mohr-Coulomb criterion with tension cut-off (for concrete).

\subsection{1 von Mises strength criterion}

Introducing the following matrices :

$$
\vec{P}=\frac{1}{3}\left[\begin{array}{cccccc}
2 & -1 & -1 & 0 & 0 & 0 \\
-1 & 2 & -1 & 0 & 0 & 0 \\
-1 & -1 & 2 & 0 & 0 & 0 \\
0 & 0 & 0 & 1 & 0 & 0 \\
0 & 0 & 0 & 0 & 1 & 0 \\
0 & 0 & 0 & 0 & 0 & 1
\end{array}\right], \quad \vec{D}=\frac{1}{2}\left[\begin{array}{cccccc}
1 & 0 & 0 & 0 & 0 & 0 \\
0 & 1 & 0 & 0 & 0 & 0 \\
0 & 0 & 1 & 0 & 0 & 0 \\
0 & 0 & 0 & 2 & 0 & 0 \\
0 & 0 & 0 & 0 & 2 & 0 \\
0 & 0 & 0 & 0 & 0 & 2
\end{array}\right]
$$

the von Mises strength criterion $\sqrt{\frac{1}{2} \underline{\underline{s}}: \underline{\underline{s}}} \leq k$, where $\underline{\underline{s}}=\underline{\underline{\sigma}}-\frac{1}{3}(\operatorname{tr} \underline{\underline{\sigma}}) \underline{\underline{1}}$ is the deviatoric stress and $k$ the ultimate shear stress, is written at each vertex $i$ of all elements as follows :

$$
\sqrt{\vec{\sigma}^{(i) T} \vec{P} \vec{D} \vec{P} \vec{\sigma}^{(i)}} \leq k_{i}
$$

where $k_{i}$ is the value of the ultimate shear stress at vertex $i$. It can also be written as:

$$
\left\|\vec{D}^{1 / 2} \vec{P} \vec{\sigma}^{(i)}\right\| \leq k_{i}
$$

which is a second-order cone (SOC) constraint. This type of constraint is particularly suited to interior point solvers such as the MosEK software package.

\subsubsection{Mohr-Coulomb strength criterion with tension cut- off}

A Mohr-Coulomb criterion with tension cut-off has been adopted to model the strength behavior of concrete :

$f(\underline{\underline{\sigma}}) \leq 0 \Longleftrightarrow\left\{\begin{array}{l}K_{p} \sigma_{I}-\sigma_{I I I} \leq f_{c} \quad \text { with } \sigma_{I} \geq \sigma_{I I} \geq \sigma_{I I I}, \quad K_{p}=\frac{1+\sin \phi}{1-\sin \phi} \\ \sigma_{I} \leq f_{t}\end{array}\right.$ 
where $f_{c}$ is the ultimate strength of concrete in compression, $f_{t}$ its tensile strength, $\phi$ the friction angle and $\sigma_{I}, \sigma_{I I}, \sigma_{I I I}$ the principal stresses.

Contrary to the von Mises criterion, this criterion is formulated in terms of principal stresses which are not known beforehand. To obtain another representation, let us introduce an auxiliary variable $y$ such that $K_{p} \sigma_{I}-\sigma_{I I I} \leq K_{p} \sigma_{I}+y \leq f_{c}$. Recalling that $\sigma_{I} \geq \sigma_{I I} \geq \sigma_{I I I}$, we then have $-\sigma_{I} \leq-\sigma_{I I} \leq-\sigma_{I I I} \leq y$. Hence, this inequality can also be written in the form of a matrix inequality : $-\vec{S} \preceq y \vec{I}_{3}$ where $\vec{S}$ is the matrix of the components of $\underline{\underline{\sigma}}$ in a given basis, $\vec{I}_{3}$ the identity matrix of size $3 \times 3$ and $\vec{X} \preceq \vec{Y}$ means that the matrix $\vec{Y}-\vec{X}$ is semi-definite positive.

Similarly, we have also that $K_{p} \sigma_{I I I}+y \leq K_{p} \sigma_{I I}+y \leq K_{p} \sigma_{I}+y \leq f_{c}$, which is equivalent to $K_{P} \vec{S}+y \vec{I}_{3} \preceq f_{c} \vec{I}_{3}$. Finally, the tension cut-off condition gives : $\sigma_{I I I} \leq \sigma_{I I} \leq \sigma_{I} \leq \vec{f}_{t}$ or also $\vec{S} \preceq f_{t} \vec{I}_{3}$.

In the end, the Mohr-Coulomb criterion with tension cut-off can be written in the following form :

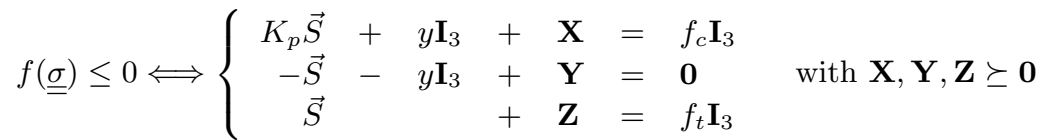

This form, which has been previously obtained in [10,11], involves linear matrix equalities and auxiliary positive semi-definite matrices $\vec{X}, \vec{Y}$ and $\vec{Z}$. These semi-definite constraints can also be accommodated using MosEK in its version 7.0 [13].

\subsection{Formulation of the optimization problem}

We can now formulate the optimization problem corresponding to the resolution of the auxiliary yield design problem. In practice, $G^{\text {hom }}$ will be obtained by finding the maximal amplification factor $\lambda^{+}$along a prescribed direction $\left(\vec{N}_{0}, \vec{M}_{0}\right)$ such that $\lambda^{+}\left(\vec{N}_{0}, \vec{M}_{0}\right) \in G^{\text {hom }}$. Hence, the corresponding optimization problem reads as :

$$
\begin{aligned}
\lambda^{+}=\max & \lambda \\
\text { s.t. } & \lambda \overrightarrow{N_{0}}-\overrightarrow{A_{N}} \boldsymbol{\Sigma}=0 \\
& \lambda \overrightarrow{M_{0}}-\overrightarrow{A_{M}} \boldsymbol{\Sigma}=0 \\
& \vec{C} \boldsymbol{\Sigma}=0 \\
& \vec{\sigma}^{(i)} \in \mathcal{K}_{i} \quad i=1, \ldots, 4 N_{E}
\end{aligned}
$$

This problem consists in optimizing a linear function of the unknowns under linear equality constraints and conic constraints at each vertex where $\mathcal{K}_{i}$ is a cone representing the strength criterion. It can be either a secondorder Lorentz cone (in the von Mises case (20)) or cones of semi-definite matrices in the case of a Mohr-Coulomb criterion (22). Hence, the problem reduces either to a SOCP problem or SDP problem depending on the strength criteria of the unit cell.

Finally, it is to be noted that since all equations are satisfied and since the criterion is satisfied inside each element (due to the linear variation of $\underline{\underline{\sigma}}$ and convexity), the maximum point $\lambda^{+}\left(\vec{N}_{0}, \vec{M}_{0}\right)$ belongs to $G^{\text {hom }}$, so that the macroscopic criterion obtained by varying the direction $\left(\vec{N}_{0}, \vec{M}_{0}\right)$ is a strict lower bound estimate for $G^{\text {hom }}$. 


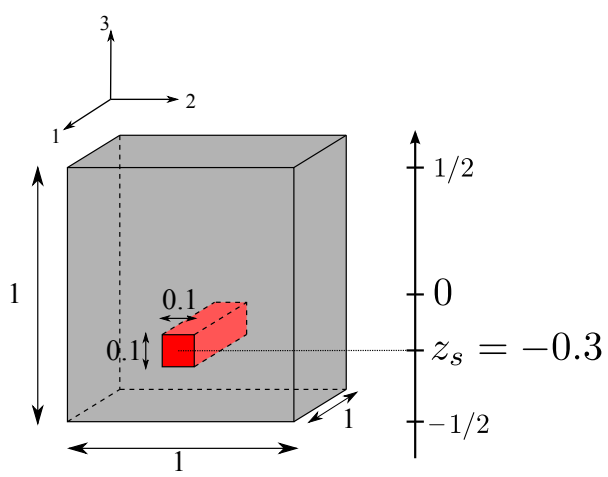

Figure 2: Unit cell geometry of the first example

\section{Illustrative applications}

\section{$4.1 \quad$ Validating example}

The first example is concerned with a cubic unit cell of unitary side length made of concrete and a steel reinforcing bar oriented in direction 1, located at a distance $z_{s}=-0.3$ below the middle plane (Figure 2). The concrete is modeled using a Mohr-Coulomb strength criterion with a friction angle of $\phi=37^{\circ}$, a compressive strength of $f_{c}=30 \mathrm{MPa}$ and a negligible tensile strength $f_{t} \approx 0$. The steel rebar is modeled using a von Mises criterion with a uniaxial strength $f_{s}=500 \mathrm{MPa}$. The volume fraction of steel is $1 \%$ (square cross section of $0.1 \times 0.1$ ).

In order to validate the computations, the strength criterion $G_{c}$ of the unreinforced unit cell (concrete only) has been computed as well as the homogenized criterion $G^{\text {hom }}$ of the reinforced unit cell. A simple analytical lower bound $G_{l b}$ of the macroscopic strength criterion can also be obtained using the fact that the volume fraction of steel is small whereas $f_{c} \ll f_{s}$. In this case, it can be proved [19] that the state of stress is almost uniaxial in the reinforcement $\underline{\underline{\sigma}}=\sigma^{s} \underline{\underline{e}}_{1} \otimes \underline{e}_{1}$ and equal to $\underline{\underline{\sigma}}^{c}$ in the concrete. The membrane force and bending moment derived from the stress state in the concrete are denoted by $\underline{N}^{c}$ and $\underline{M}^{c}$. Then, the total membrane force and bending moment are given by :

$$
\begin{array}{r}
\underline{\underline{N}}=\underline{\underline{N^{c}}}+A_{s} \sigma^{s} \underline{e}_{1} \otimes \underline{e}_{1} \\
\underline{\underline{M}}=\underline{\underline{M}}^{c}-A_{s} z_{s} \sigma^{s} \underline{e}_{1} \otimes \underline{e}_{1}
\end{array}
$$

where $A_{s}$ is the section of the steel bar. Now, if $\underline{\sigma}^{c} \in \mathcal{G}^{c}$ satisfies the local concrete strength criterion and $\left|\sigma^{s}\right| \leq f_{s}$, then $(\underline{\underline{N}}, \underline{\underline{M}}) \in G^{\text {hom }}$. Therefore, denoting by $G^{c}$ the concrete strength criterion expressed in terms of generalized forces, we have the following lower bound estimate for $G^{\text {hom }}$ :

$$
G_{l b}=G^{c} \oplus L^{s} \subseteq G^{h o m}
$$

where $L^{s}=\left\{\left(N_{s} \underline{e}_{1} \otimes \underline{e}_{1},-z_{s} N_{s} \underline{e}_{1} \otimes \underline{e}_{1}\right) ;\left|N_{s}\right| \leq N_{y}=A_{s} f_{s}\right\}$. This construction means that the lower bound approach is obtained by translating the criterion of the concrete only along the generalized vector $\pm N_{y}\left(\underline{e}_{1} \otimes \underline{e}_{1},-z_{s} \underline{e}_{1} \otimes \underline{e}_{1}\right)$. A kinematic approach would show that this simple construction coincides with $G^{\text {hom }}$ in the limit of infinitely small 


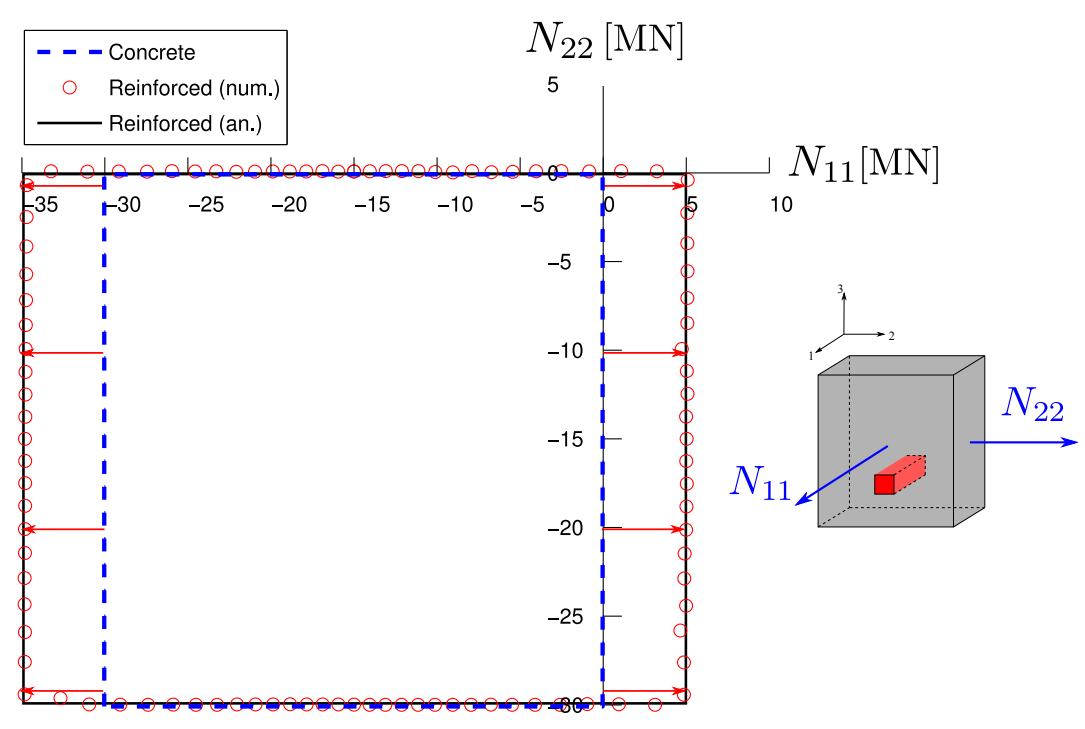

Figure 3: Projections on the plane $N_{11}-N_{22}$

volume fraction of steel and infinite contrast between steel and concrete resistances.

First, the projections of the different criteria onto the plane $N_{11}-N_{22}$ of membrane forces in directions 1 and 2 are represented in Figure 3. Hence, all components of $\underline{M}$ as well as the $N_{12}$ component are left as free optimization variables. The criterion for concrete only $G^{c}$ (blue dashed lines) corresponds to a square in the compression region only because of the fact that the tensile strength has been neglected. The inclusion of a reinforcement improves both traction and compression strengths in the $N_{11}$ direction but not in direction 2, as expected. Furthermore, the numerically obtained macroscopic strength criterion (red circles) perfectly matches the analytical lower bound (black solid lines) which is obtained by translating the criterion of concrete by the previously mentioned vectors here drawn in red.

In Figure 4, the axial-bending interaction diagram along the direction 1 has been represented (all $N_{i j}=M_{i j}=0$ except for $i=j=1$ ). Here, the classical result of the criterion for concrete only, consisting of two parabolas, is retrieved. The effect of the reinforcement expands the interaction diagram in a direction along vectors $\pm(1 \mathrm{MN}, 0.3 \mathrm{MN} . \mathrm{m})$ as confirmed by the analytical lower bound.

\subsection{Fire resistance of reinforced concrete slabs}

The second illustrative application is concerned with the fire resistance of reinforced concrete (RC) slabs. Experiments have been conducted by the French Scientific and Technical Center for Building and were aimed at studying the evolution of strength properties (uniaxial bending capacity in a first step) of RC slabs in fire conditions. Four-points bending tests 


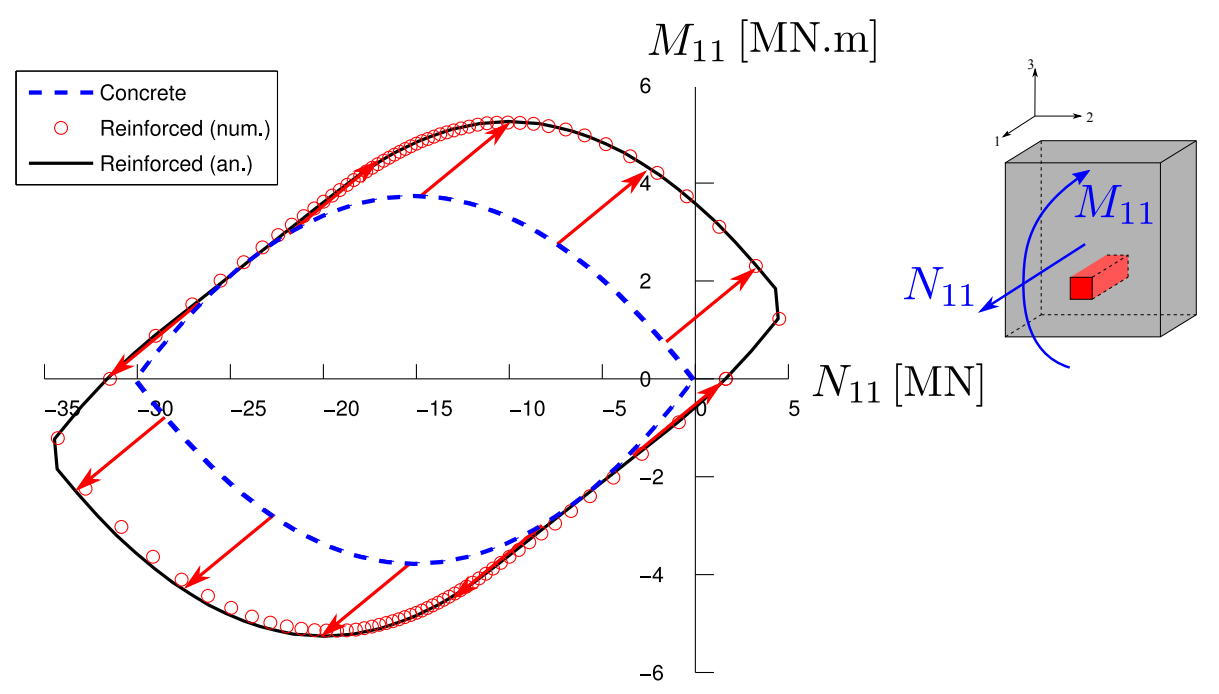

Figure 4: $N_{11}-M_{11}$ interaction diagram

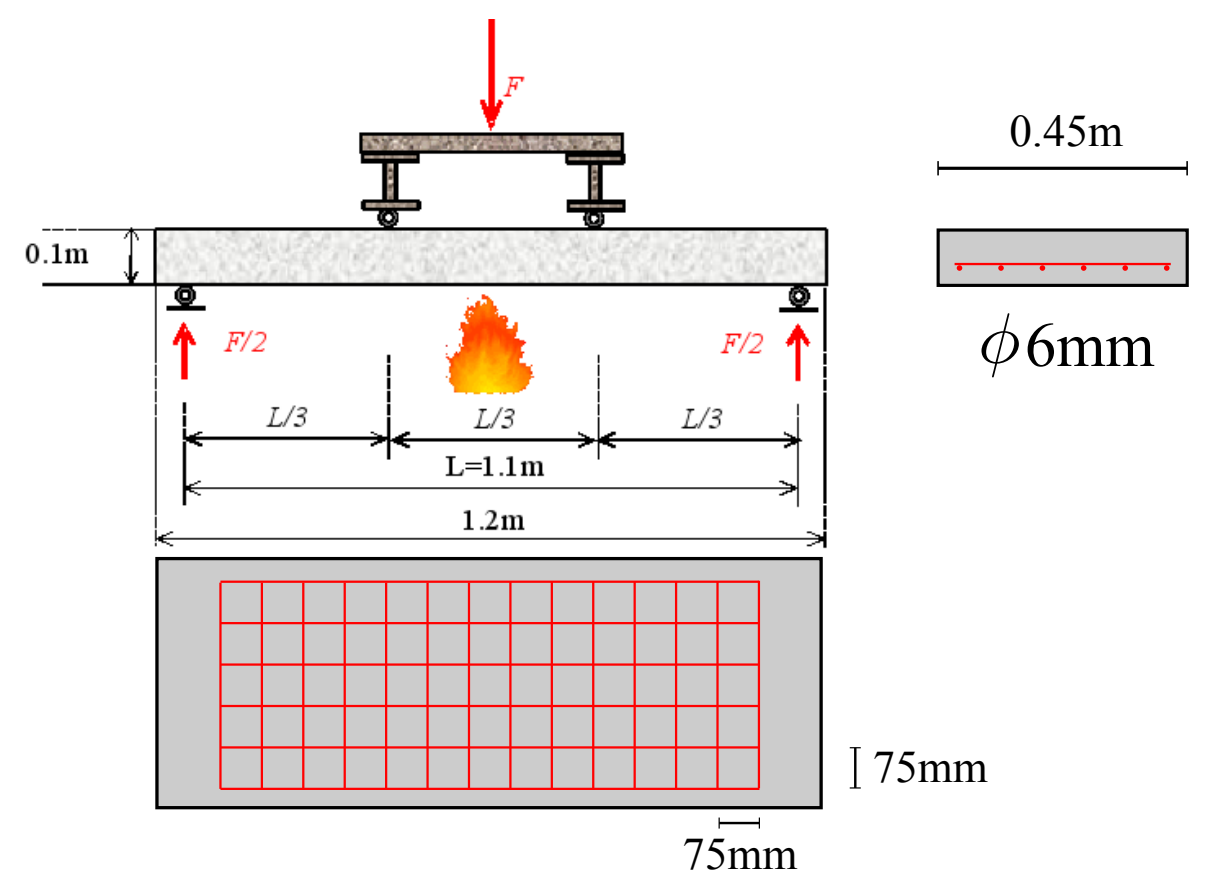

Figure 5: Experimental setup of 4-points bending tests in fire conditions and RC slabs geometry 

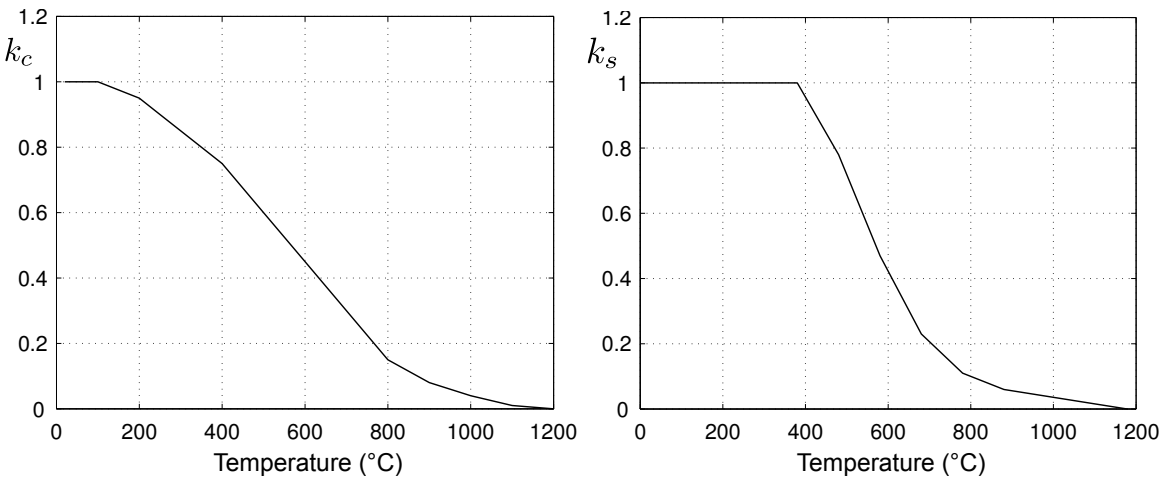

Figure 6: Reduction factors for concrete (left) and steel (right) as a function of temperature

have been performed on $\mathrm{RC}$ slabs previously placed in an oven which heated the bottom surface of the RC slabs (Figure 5). At a given stabilized temperature inside the oven and temperature gradient across the plate thickness, four-points bending tests have been performed up to failure. The aim of this example is to compare these experimental results to numerical computations using the previously described approach.

\subsubsection{Computations in fire conditions}

The general principle of the computations in fire conditions is rather straightforward. The strength characteristics of concrete and steel at ambient temperature are known from characterization experiments and thermocouples have been placed at various heights in the plate thickness during the tests so as to measure the temperature distribution through the thickness during the experiment.

The key point concerns the degradation of the strength properties of concrete and steel with the temperature. These strength properties introduced in the numerical simulations have been decreased according to Eurocodes prescriptions providing so-called reduction factors $k_{c}(\Theta)$ and $k_{s}(\Theta)$ which are decreasing functions of the temperature $\Theta$, so that $f_{c}(\Theta)=k_{c}(\Theta) f_{c, a m b}$ and $f_{s}(\Theta)=k_{s}(\Theta) f_{s, a m b}$ where $f_{c, a m b}\left(\right.$ resp. $\left.f_{s, a m b}\right)$ is the compressive strength of concrete (resp. uniaxial strength of steel) at ambient temperature. For the materials used in this experiment, such reduction factors are represented in Figure 6. The concrete friction angle is assumed to remain unaffected by the temperature increase.

Therefore, to a given temperature profile across the slab thickness, is associated a non-uniform distribution of strength properties in the plate thickness. Yield design computations have then been performed on this unit cell with these non-uniform distributions so as to obtain a macroscopic strength criterion depending on the temperature field : $G^{h o m}(\Theta(z))$.

\subsubsection{Numerical results}

We performed computations on the unit cell represented in Figure 7 with the 4 different temperature profiles represented in Figure 8. The corre- 


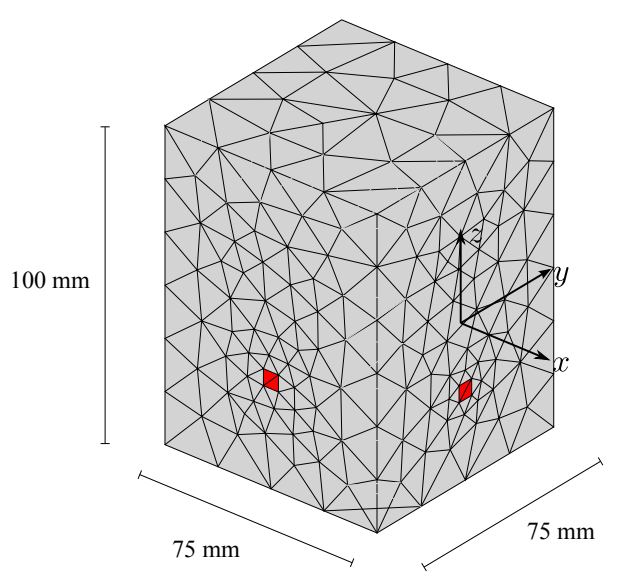

Figure 7: Unit cell geometry and mesh for the second example

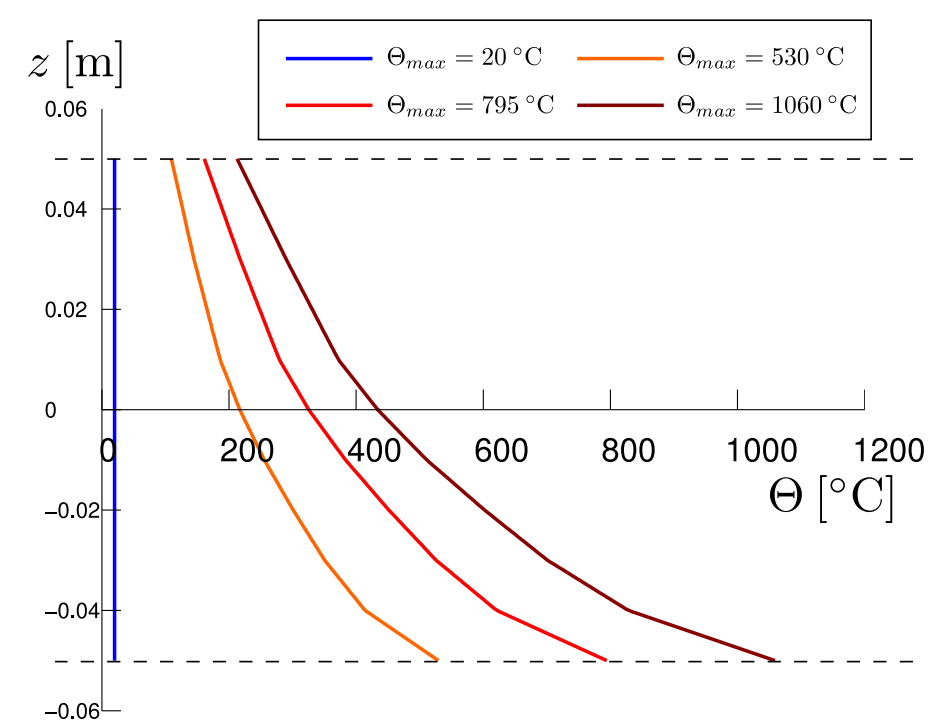

Figure 8: Temperature fields used in the computations 


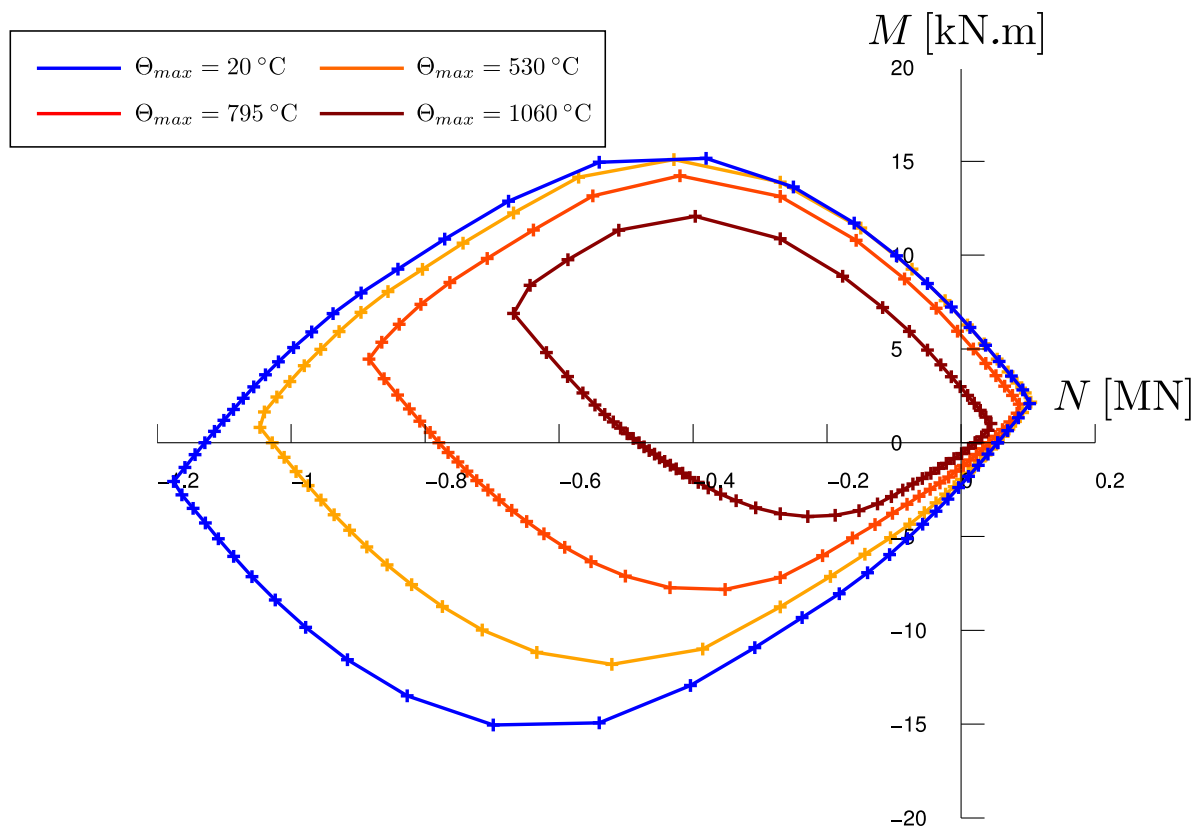

Figure 9: Evolution of the $N_{11}-M_{11}$ interaction diagram with respect to the temperature

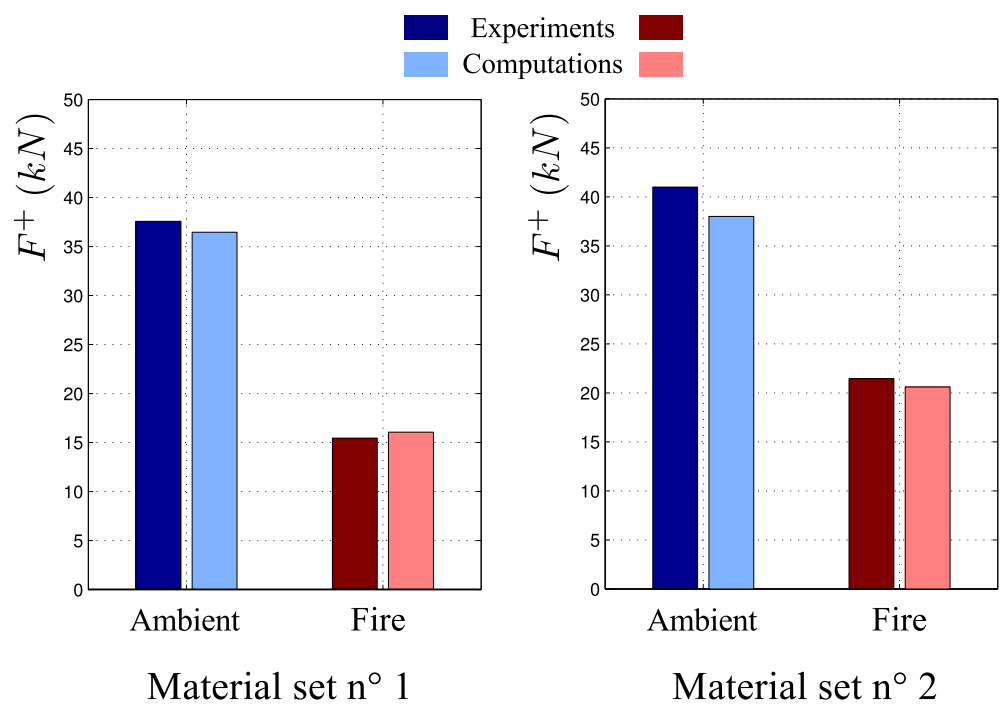

Figure 10: Comparison of ultimate loads obtained from the experiments and numerical computations 
sponding $N_{11}-M_{11}$ interaction diagrams have been represented in Figure 9. It can clearly be observed that as the temperature increases, the interaction diagram reduces in size because of the reduction of all strength properties. Besides, it also "rotates" due to the fact that, owing to the temperature gradient, the lower part of the slab becomes weaker than the upper part.

\subsubsection{Confrontation to experimental results}

In the experiments, two sets of materials for steel and concrete have been considered and 4-points bending tests have been performed until failure at ambient temperature as well as at an oven temperature approaching $1000{ }^{\circ} \mathrm{C}$. The temperature distribution in such fire conditions is close to the one represented in Figure 8 for $\Theta_{\max }=1060{ }^{\circ} \mathrm{C}$. The choice of a 4 -point bending test enables to relate directly the ultimate load at failure to the ultimate pure bending moment in the direction of the slab, since the bending moment is constant in the middle span of the structure. Hence, ultimate loads obtained from the experiments have been compared to those obtained from numerical computations of the ultimate bending moment. These results are reported in Figure 10 for ambient and fire conditions and for the two different material sets. It can be seen that the numerical predictions, labeled in light blue and light red, compare very well to the ultimate loads measured in the experiments labeled in dark blue and dark red.

\section{Conclusions}

A homogenization procedure for assessing the strength properties of periodically heterogeneous plates has been presented in the framework of yield design theory. The present work more specifically focuses on the case when the typical length of the plate heterogeneities and the plate thickness are of the same order. In this case, the unit cell is modeled as a 3D body on which an auxiliary yield design problem is formulated, the resolution of which enables to compute the macroscopic strength domain. A lower bound static approach using 3D equilibrium finite elements is presented and a conic optimization problem is formulated. Finally, the proposed method is applied to reinforced concrete slabs in fire conditions. It allows to predict, for instance, the evolution of interaction diagrams with respect to different temperature fields. These results are also confronted to ultimate loads obtained from experiments carried out in fire conditions.

It would be interesting to complete this work by an upper bound kinematic approach on the same problem, so as to bracket the exact macroscopic strength criterion, since the present method only yields lower bounds. Besides, further work could also investigate the use of such criteria in global computations on a complex structure.

\section{References}

[1] P. Suquet, Homogenization techniques for composite media 272, 193 (1985)

[2] P. de Buhan, A fundamental approach to the yield design of reinforced soil structures. Ph.D. thesis, Thèse d'Etat, Paris VI (1986) 
[3] S. Maghous, Détermination du critère de résistance macroscopique d'un matériau hétérogène à structure périodique. Approche numérique. These, Ecole Nationale des Ponts et Chaussées (1991). URL http://tel.archives-ouvertes.fr/tel-00529369

[4] J.J. Marigo, P. Mialon, J.C. Michel, P. Suquet, Journal de Mécanique théorique et appliquée 6(1), 47 (1987)

[5] J. Pastor, S. Turgeman, J. de Mécanique Théorique et Appliquée 2, 393 (1983)

[6] S. Turgeman, J. Pastor, Journal de mécanique théorique et appliquée 6(1), 121 (1987)

[7] P. Francescato, J. Pastor, Revue Européenne des Éléments Finis 7(4), 421 (1998)

[8] K. Sab, Comptes Rendus Mecanique 331(9), 641 (2003)

[9] G. Hassen, M. Gueguin, P. de Buhan, European Journal of Mechanics - A/Solids 37(0), 266 (2013). DOI 10.1016/j.euromechsol.2012.07.003

[10] C. Bisbos, P. Pardalos, Journal of Optimization Theory and Applications 134(2), 275 (2007)

[11] K. Krabbenhøft, A. Lyamin, S. Sloan, International Journal of Solids and Structures 44(5), 1533 (2007)

[12] A. Makrodimopoulos, International Journal for Numerical Methods in Biomedical Engineering 26(11), 1449 (2010)

[13] Mosek. The Mosek optimization toolbox for MATLAB manual (2008). URL http://www.mosek.com/

[14] J. Salençon, Calcul à la rupture et analyse limite (Presses de l'Ecole Nationale des Ponts et Chaussées, 1983)

[15] J. Salençon, Yield Design (Wiley. com, 2013)

[16] J. Bleyer, P. de Buhan, International Journal of Solids and Structures in press, 28 (2014). DOI http://dx.doi.org/10.1016/j.ijsolstr.2014.03.018

[17] S. Bourgeois, Modélisation numérique des panneaux structuraux légers. Ph.D. thesis, Université d'Aix-Marseille 2, FRANCE (1997)

[18] J. Dallot, K. Sab, Journal of the Mechanics and Physics of Solids 56(2), 561 (2008)

[19] P. de Buhan, A. Taliercio, European Journal of Mechanics - A/Solids 10(2), 129 (1991) 